\title{
Black Holes Studied as a Population
}

\author{
The latest dataset from gravitational-wave observatories has enough \\ events to allow researchers to study properties of the whole population of \\ black holes.
}

By David Ehrenstein

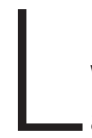

ess than six years after the first detection of gravitational waves, observations are becoming routine, with LIGO and Virgo logging black hole mergers more than once per week. At the APS April meeting, the LIGO-Virgo Collaboration (LVC) reported using their catalog of nearly 50 events to estimate the typical properties and histories of black holes. Measurements of black hole spins, for example, suggest that at least two different formation mechanisms are common for black hole binaries. These black hole "population" studies-akin to astronomers' star surveys-are becoming a prized tool for gravitational-wave scientists, in addition to

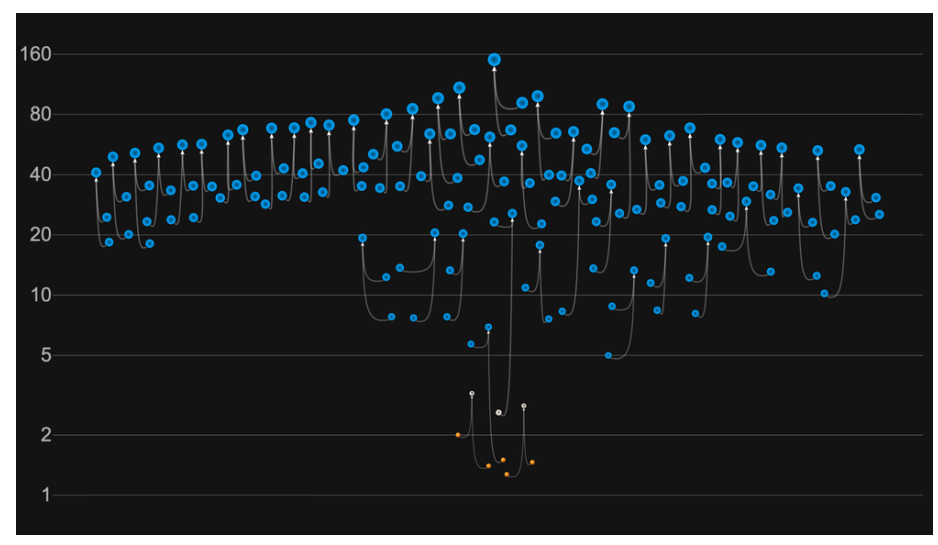

Black holes (blue), neutron stars (orange), and compact objects of uncertain nature (gray) detected via gravitational waves through September 2019. Each binary merger involves three compact objects: the two coalescing objects and the final remnant. The vertical scale is in solar masses.

Credit: F. Elavsky and A. Geller/Northwestern Univ./LIGO-Virgo Collaboration studies of individual events.

The black holes in the LVC catalog are stellar-mass black holes-the remnants of giant stars after they explode as supernovae. In the past, astronomers could spot these black holes only when they were in a binary orbit with a normal star, but the LIGO and Virgo observatories have revolutionized the field since 2015. "The vast majority of the stellar-mass black holes that we know about in the Universe [were detected via] gravitational waves," said Carl Rodriguez of Carnegie Mellon University, Pennsylvania, in his presentation at the conference. So gravitational waves are now the main source of data from which astrophysicists will learn about these objects. "For the first time, we're able to do astronomy" using gravitational waves, said Maya Fishbach of Northwestern University, Illinois, a member of the LVC. "We're really going to learn more about star formation in general."

In his talk, LVC member Thomas Dent, of the University of Santiago de Compostela in Spain, presented several results based on analyses of 47 events. He showed the mass distribution-a plot giving the rate of mergers vs the primary (larger) mass of a merging black hole pair. The rate decreases with increasing mass, and the curve can be mathematically described as having a small bump at about 33 solar masses, although the shape is not yet completely certain. This feature reflects the fact that there is an excess of mergers with primary masses in the range of about 30-40 solar masses and then a sudden decrease at higher masses.

These characteristics of the mass spectrum are not yet very well understood, Dent said. A so-called pair-instability supernova 


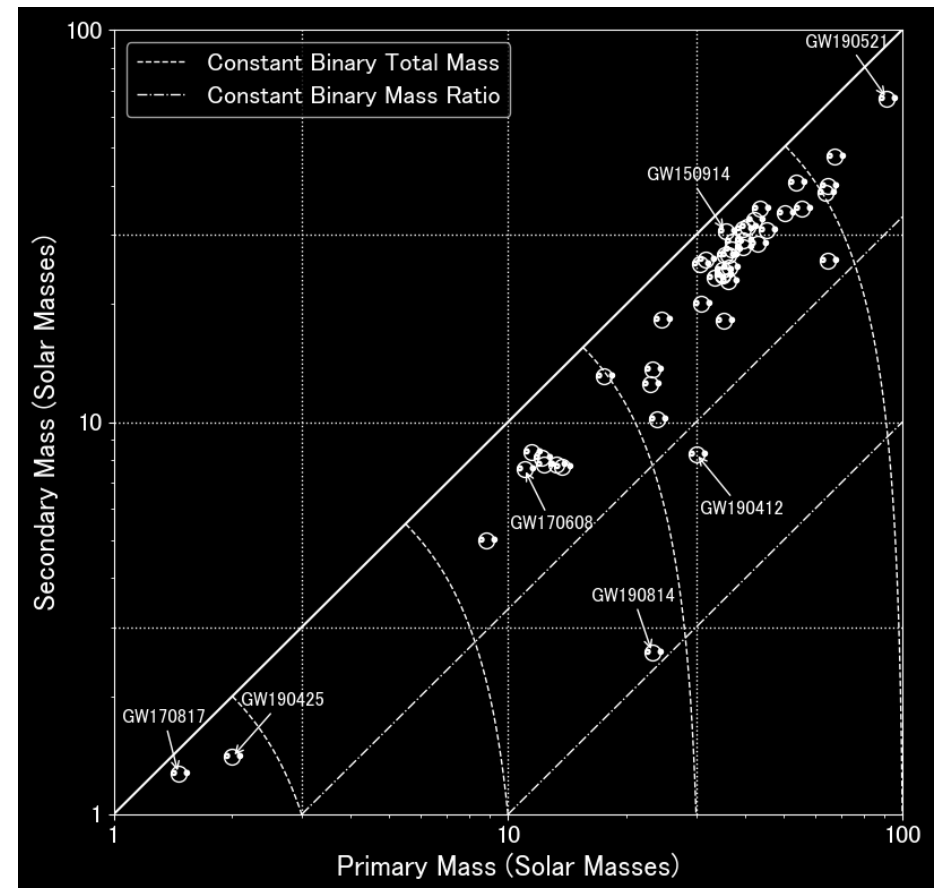

Masses of the binary objects for merger events observed through September 2019. The data show an unexpected concentration of primary black holes at around 33 solar masses (at the position of GW150914-the first detected gravitational-wave event). There are also a surprising number of black holes in a predicted gap between 45 and 120 solar masses. (The seemingly small number of primary black holes weighing less than 30 solar masses is a selection effect, as mergers of smaller objects produce a weaker, harder-to-detect signal than those of larger objects.)

Credit: T. Dent/IGFAE/LIGO-Virgo Collaboration

(PISN) - a type associated with the largest stars - is expected to leave behind either a small black hole or no black hole at all, producing a gap in the spectrum between about 45 and 120 solar masses. But the latest catalog, comprising data through September 2019, includes several black holes that weigh up to 85 solar masses. Furthermore, a "pile-up" of black holes just below 45 solar masses was also expected, resulting from a process by which stars at the lower end of the PISN range lose mass and produce more typical supernovae. However, the observed bump is at 33 solar masses, too low to fit the conventional theory. The models could be adjusted somewhat, Dent said, but there's no model currently that can accommodate both the low-mass bump and the higher-mass black holes in the predicted gap.

Dent also discussed the collaboration's results on black hole spins and what they mean for the histories of black hole binaries. Researchers often discuss two types of black-hole-binary formation mechanisms: isolated and dynamic. Isolated black hole binaries form from binary star systems in which both stars die and leave black holes behind. Dynamic binaries form in star-dense environments like globular clusters when two solitary black holes meet and become gravitationally bound.

Isolated binaries are expected to have the spin axes of the two black holes relatively well aligned with the orbital axis that defines their motion around each other, and their spins should be in the same direction as their orbits (clockwise or counterclockwise). But the spin axes and rotation directions of a dynamic pair should be uncorrelated because they were spinning long before they met. The latest catalog data show a broad distribution of spin-orbital alignment, including between 12 and $44 \%$ of the events having one or both spins antialigned with their orbital motion. If the spins were entirely uncorrelated, this fraction would be $50 \%$. The fact that the antialigned fraction is significant-but less than half-strongly suggests that both formation mechanisms must be at work, Dent said.

David Ehrenstein is a Senior Editor for Physics. 